\title{
Polar duals of convex and star bodies
}

\author{
Chang-Jian Zhao $^{1 *}$, Lian-Ying Chen ${ }^{1}$ and Wing-Sum Cheung ${ }^{2}$
}

\footnotetext{
* Correspondence: chjzhao@yahoo. com.cn

'Department of Mathematics, China Jiliang University, Hangzhou 310018, China

Full list of author information is available at the end of the article
}

\begin{abstract}
In this article, some new inequalities about polar duals of convex and star bodies are established. The new inequalities in special case yield some of the recent results.
\end{abstract}

MR (2000) Subject Classification: 52A30.

Keywords: polar dual, $L_{p}$-mixed volume, dual $L_{p}$-mixed volume, the Bourgain and Milman's inequality

\section{Notations and preliminaries}

The setting for this article is $n$-dimensional Euclidean space $\mathbb{R}^{n}(n>2)$. Let $\mathcal{K}^{n}$ denotes the set of convex bodies (compact, convex subsets with non-empty interiors) in $\mathbb{R}^{n}$. We reserve the letter $u$ for unit vectors, and the letter $B$ for the unit ball centered at the origin. The surface of $B$ is $S^{n-1}$. The volume of the unit $n$-ball is denoted by $\omega_{n}$.

We use $V(K)$ for the $n$-dimensional volume of convex body $K \cdot h(K, \cdot): S^{n-1} \rightarrow \mathbb{R}$, denotes the support function of $K \in \mathcal{K}^{n}$; i.e., for $u\left\lfloor S^{n-1}\right.$

$$
h(K, u)=\operatorname{Max}\{u \cdot x: x \in K\},
$$

where $u \cdot x$ denotes the usual inner product $u$ and $x$ in $\mathbb{R}^{n}$.

Let $\delta$ denotes the Hausdorff metric on $\mathcal{K}^{n}$, i.e., for $K, L \in \mathcal{K}^{n}, \delta(K, L)=\left|h_{K}-h_{L}\right|_{\infty}$, where $|\cdot|_{\infty}$ denotes the sup-norm on the space of continuous functions $C\left(S^{n-1}\right)$.

Associated with a compact subset $K$ of $\mathbb{R}^{n}$, which is star-shaped with respect to the origin, is its radial function $\rho(K, \cdot): S^{n-1} \rightarrow \mathbb{R}$, defined for $u L S^{n-1}$, by

$$
\rho(K, u)=\operatorname{Max}\{\lambda \geq 0: \lambda u \in K\} .
$$

If $\rho(K, \cdot)$ is positive and continuous, $K$ will be called a star body. Let $S^{n}$ denotes the set of star bodies in $\mathbb{R}^{n}$. Let $\tilde{\delta}$ denotes the radial Hausdorff metric, as follows, if $K, L$ $S^{n}$, then $\tilde{\delta}(K, L)=\left|\rho_{K}-\rho_{L}\right|_{\infty}($ See $[1,2])$.

\section{1 $L_{p}$-mixed volume and dual $L_{p}$-mixed volume}

If $K, L \in \mathcal{K}^{n}$, the $L_{p}$-mixed volume $V_{p}(K, L)$ was defined by Lutwak (see [3]):

$$
V_{p}(K, L)=\frac{1}{n} \int_{S^{n-1}} h(L, u)^{p} \mathrm{~d} S_{p}(K, u),
$$

where $S_{p}(K, \cdot)$ denotes a positive Borel measure on $S^{n-1}$.

The $L_{p}$ analog of the classical Minkowski inequality (see [3]) states that: If $K$ and $L$ are convex bodies, then

(c) 2012 Zhao et al; licensee Springer. This is an Open Access article distributed under the terms of the Creative Commons Attribution License (http://creativecommons.org/licenses/by/2.0), which permits unrestricted use, distribution, and reproduction in any medium, provided the original work is properly cited. 


$$
V_{p}(K, L) \geq V(K)^{(n-p) / n} V(L)^{p / n},
$$

with equality if and only if $K$ and $L$ are homothetic.

If $K, L \in S^{n}, p \geq 1$, the $L_{p}$-dual mixed volume $\tilde{V}_{-p}(K, L)$ was defined by Lutwak (see $[4])$ :

$$
\tilde{V}_{-p}(K, L)=\frac{1}{n} \int_{S^{n-1}} \rho(K, u)^{n+p} \rho(L, u)^{-p} \mathrm{~d} S(u),
$$

where $d S(u)$ signifies the surface area element on $S^{n-1}$ at $u$.

The following dual $L_{p}$-Minkowski inequality was obtained in [2]: If $K$ and $L$ are star bodies, then

$$
\tilde{V}_{-p}(K, L)^{n} \geq V(K)^{n+p} V(L)^{-p},
$$

with equality if and only if $K$ and $L$ are dilates.

\subsection{Mixed bodies of convex bodies}

If $K_{1}, \ldots, K_{n-1} \in \mathcal{K}^{n}$, the notation of mixed body $\left[K_{1}, \ldots, K_{n-1}\right]$ states that (see [5]): corresponding to the convex bodies $K_{1}, \ldots, K_{n-1} \in \mathcal{K}^{n}$ in $\mathbb{R}^{n}$, there exists a convex body, unique up to translation, which we denote $b y\left[K_{1}, \ldots, K_{n-1}\right]$.

The following is a list of the properties of mixed body: It is symmetric, linear with respect to Minkowski linear combinations, positively homogeneous, and for $K_{i} \in \mathcal{K}^{n}, i=1, \ldots, n, L_{1} \in \mathcal{K}^{n}$ and $\lambda_{\mathrm{i}}>0$,

(1) $V_{1}\left(\left[K_{1}, \ldots, K_{n-1}\right], K_{n}\right)=V\left(K_{1}, \ldots, K_{n-1}, K_{n}\right)$;

(2) $\left[K_{1}+\mathrm{L}_{1}, K_{2}, \ldots, K_{n-1}\right]=\left[K_{1}, K_{2}, \ldots, K_{n-1}\right]+\left[L_{1}, K_{2}, \ldots, K_{n-1}\right]$;

(3) $\left[\lambda_{1} K_{1}, \ldots, \lambda_{n-1} K_{n-1}\right]=\lambda_{1} \ldots \lambda_{n-1} \cdot\left[K_{1}, \ldots, K_{n-1}\right]$;

(4) $\underbrace{[K, \ldots, K]}_{n-1}=K$.

The properties of mixed body play an important role in proving our main results.

\subsection{Polar of convex body}

For $K \in \mathcal{K}^{n}$, the polar body of $K, K^{*}$ is defined:

$$
K^{*}=\left\{x \in \mathbb{R}^{n}: x \cdot y \leq 1, y \in K\right\} .
$$

It is easy to get that

$$
\rho(K, u)^{-1}=h\left(K^{*}, u\right) .
$$

Bourgain and Milman's inequality is stated as follows (see [6]).

If $K$ is a convex symmetric body in $\mathbb{R}^{n}$, then there exists a universal constant $c>0$ such that

$$
V(K) V\left(K^{*}\right) \geq c^{n} \omega_{n}^{2} .
$$

Different proofs were given by Pisier [7]. 


\section{Main results}

In this article, we establish some new inequalities on polar duals of convex and star bodies.

Theorem 2.1 If $K, K_{1}, \ldots, K_{n-1}$ are convex bodies in $\mathbb{R}^{n}$ and let $L=\left[K_{1}, \ldots, K_{n-1}\right]$, then the $L_{p}$-mixed volumes $V_{p}(K, L), V_{p}\left(K^{*}, L\right), V_{p}(B, L)$ satisfy

$$
V_{p}(K, L) V_{p}\left(K^{*}, L\right) \geq V_{p}(B, L)^{2} .
$$

Proof From (1.1) and (1.2), it is easy

$$
h(K, u) \geq \rho(K, u), \quad K \in \mathcal{K}^{n} .
$$

By definition of $L_{p}$-mixed volume, we have

$$
V_{p}(K, L)=\frac{1}{n} \int_{S^{n-1}} h(K, u)^{p} \mathrm{~d} S_{p}(L ; u),
$$

and

$$
V_{p}\left(K^{*}, L\right)=\frac{1}{n} \int_{S^{n-1}} h\left(K^{*}, u\right)^{p} \mathrm{~d} S_{p}(L, u) .
$$

Multiply both sides of (2.3) and (2.4), in view of (1.7) and (2.2) and using the Cauchy-Schwarz inequality (see [8]), we obtain

$$
\begin{aligned}
n^{2} & V_{p}(K, L) V_{p}\left(K^{*}, L\right) \\
& =\left(\int_{S^{n-1}} h(K, u)^{p} \mathrm{~d} S_{p}\left(K_{1}, \ldots, K_{n-1} ; u\right)\right)\left(\int_{S^{n-1}} \frac{1}{\rho(K, u)^{p}} \mathrm{~d} S_{p}\left(K_{1}, \ldots, K_{n-1} ; u\right)\right) \\
& \geq\left(\int_{S^{n-1}} h(K, u)^{\frac{p}{2}} \cdot \frac{1}{\rho(K, u)^{\frac{p}{2}}} \mathrm{~d} S_{p}\left(K_{1}, \ldots, K_{n-1} ; u\right)\right)^{2} \\
& \geq\left(\int_{S^{n-1}} \mathrm{~d} S_{p}\left(K_{1}, \ldots, K_{n-1} ; u\right)\right)^{2} \\
& =n^{2} V_{p}^{2}(B, L) .
\end{aligned}
$$

Taking $p=n-1$ in (2.1) and in view of the property (1) of mixed body, we obtain the following result: If $K, K_{1}, \ldots, K_{n-1} \in \mathcal{K}^{n}$, then

$$
V\left(K, K_{1}, \ldots, K_{n-1}\right) V\left(K^{*}, K_{1}, \ldots, K_{n}\right) \geq V\left(B, K_{1}, \ldots, K_{n-1}\right)^{2} .
$$

This is just an inequality given by Ghandehari [9].

Let $L=B$, we have the following interesting result:

Let $K$ be a convex body and $K^{*}$ its polar dual, then

$$
V_{p}(K, B) V_{p}\left(K^{*}, B\right) \geq \omega_{n}^{2} .
$$

Taking $p=n-1$ in (2.6), we have the following result which was given in [9]:

$$
W_{n-1}(K) W_{n-1}\left(K^{*}\right) \geq \omega_{n}^{2}
$$


with equality if and only if $K$ is an $n$-ball.

Corollary 2.2 The $L_{p}$-mixed volume of $K$ and $K^{*}, V_{p}\left(K, K^{*}\right)$ satisfies

$$
V_{p}\left(K^{*}, K\right)^{n} \geq \omega_{n}^{2(n-p)} V(K)^{2 p-n} .
$$

Proof In view of the property (4) of the mixed body, we have

$$
V_{p}(K,[K, \ldots, K])=V_{p}(K, K)=V(K) .
$$

Form (1.4) and taking for $K_{1}=K_{2}=\ldots=K_{n-1}=K$ in (2.1), we have

$$
\begin{aligned}
V(K) V_{p}\left(K^{*}, K\right) & \geq V_{p}^{2}(B, K) \\
& \geq V(B) \frac{2(n-p)}{n} V(K) \frac{2 p}{n} \\
& =\omega_{n} \frac{2(n-p)}{n} V(K)^{\frac{2 p}{n}} .
\end{aligned}
$$

Taking $p=n-1$ in (2.7), we have the following result:

$$
V(K^{*}, \underbrace{K, \ldots, K}_{n-1})^{n} \geq \omega_{n}^{2} V(K)^{n-2} .
$$

This is just an inequality given by Ghandehari [9]. The cases $p=1$ and $n=2$ give Steinhardt's and Firey's result (see [7]).

A reverse inequality about $\tilde{V}(K^{*}, \underbrace{K, \ldots, K}_{n-1})$ was given by Ghandehari [9].

$$
\tilde{V}(K^{*}, \underbrace{K, \ldots, K}_{n-1})^{n} \leq \omega_{n}^{2} V(K)^{n-2} .
$$

Theorem 2.3 Let $K$ be a star body in $\mathbb{R}^{n}, K^{*}$ be the polar dual of $K$, then there exist a universal constant $c>0$ such that

$$
V(K)^{n+2 p} \tilde{V}_{-p}\left(K^{*}, K\right)^{n} \geq\left(c^{n} \omega_{n}^{2}\right)^{n+p},
$$

where $c$ is the constant of Bourgain and Milman's inequality.

Proof From (1.6) and (1.8), we have

$$
\begin{aligned}
\tilde{V}_{-p}\left(K^{*}, K\right) & \geq V\left(K^{*}\right)^{\frac{n+p}{n}} V(K)^{-\frac{p}{n}} \\
& =\left(V\left(K^{*}\right) V(K)\right)^{\frac{n+p}{n}} V(K)^{-\frac{n+2 p}{n}} \\
& \geq\left(c^{n} \omega_{n}^{2}\right)^{\frac{n+p}{n}} V(K)^{-\frac{n+2 p}{n}} .
\end{aligned}
$$

The following theorem concerning $L_{p}$-dual mixed volumes will generalize Santaló inequality.

Theorem 2.4 Let $K_{1}$ and $K_{2}$ be two star bodies, $K_{1}^{*}$ and $K_{2}^{*}$ be the polar dual of $K_{1}$ and $K_{2}$, then there exists a constant $c, L_{p}$-dual mixed volumes $\tilde{V}_{-p}\left(K_{1}, K_{2}\right)$ and $\tilde{V}_{-p}\left(K_{1}, K_{2}\right) \tilde{V}_{-p}\left(K_{1}^{*}, K_{2}^{*}\right) \geq c^{n} \omega_{n}^{2}$ satisfy

$$
\tilde{V}_{-p}\left(K_{1}, K_{2}\right) \tilde{V}_{-p}\left(K_{1}^{*}, K_{2}^{*}\right) \geq c^{n} \omega_{n}^{2} .
$$


Proof From (1.6), we have

$$
\tilde{V}_{-p}\left(K_{1}, K_{2}\right) \geq\left(K_{1}\right)^{\frac{n+p}{n}} V\left(K_{2}\right)^{-\frac{p}{n}} .
$$

For $K_{1}^{*}$ and $K_{2}^{*}$, we also have

$$
\tilde{V}_{-p}\left(K_{1}^{*}, K_{2}^{*}\right) \geq V\left(K_{1}^{*}\right) \frac{n+p}{n} V\left(K_{2}^{*}\right)-\frac{p}{n} .
$$

Multiply both sides of (2.10) and (2.11) and using Bourgain and Milman's inequality, we obtain

$$
\begin{aligned}
\tilde{V}_{-p}\left(K_{1}, K_{2}\right) \tilde{V}_{-p}\left(K_{1}^{*}, K_{2}^{*}\right) & \geq\left(V\left(K_{1}\right) V\left(K_{1}^{*}\right)\right)^{-\frac{p}{n}}\left(V\left(K_{2}\right) V\left(K_{2}^{*}\right)\right)^{-\frac{p}{n}} \\
& \geq\left(c^{n} \omega_{n}^{2}\right)^{\frac{n+p}{n}}\left(c^{n} \omega_{n}^{2}\right)^{-\frac{p}{n}} \\
& =c^{n} \omega_{n}^{2} .
\end{aligned}
$$

Taking for $K_{1}=K_{2}=K$ in (2.9) and in view of $\tilde{V}_{-p}\left(K_{1}, K_{2}\right)=\tilde{V}_{-p}(K, K)=V(K),(2.9)$ changes to the Bourgain and Milman's inequality (1.8).

\section{Acknowledgements}

C.-J. Zhao research was supported by National Natural Sciences Foundation of China (10971205). W.-S. Cheung research was partially supported by a HKU URG grant.

\section{Author details}

'Department of Mathematics, China Jiliang University, Hangzhou 310018, China 2Department of Mathematics, The University of Hong Kong, Pokfulam Road, Hong Kong

\section{Authors' contributions}

C-JZ, L-YC and W-SC jointly contributed to the main results Theorems 2.1, 2.3, and 2.4. All authors read and approved the final manuscript.

\section{Competing interests}

The authors declare that they have no competing interests.

Received: 17 December 2011 Accepted: 17 April 2012 Published: 17 April 2012

\section{References}

1. Schneider, R: Convex Boides: The Brunn-Minkowski Theory. Cambridge University Press Cambridge (1993)

2. Gardner, RJ: Geometric Tomography. Cambridge University Press New York (1996)

3. Lutwak, E: The Brunn-Minkowski-Firey theory-l: mixed volumes and the Minkowski problem. J Diff Geom. 38, 131-150 (1993)

4. Lutwak, E, Yang, D, Zhang, GY: L affine isoperimetric inequalities. J Diff Geom, 56, 111-132 (2000)

5. Lutwak, E: Volume of mixed bodies. Trans Am Math Soc. 294, 487-500 (1986)

6. Bourgain, J, Milman, V: New volume ratio properties for convex symmetric bodies in [ineq]. Invent Math. 88, 319-340 (1987)

7. Pisier, G: The volume of convex bodies and Banach space geomery. Cambridge University Press Cambridge (1989)

8. Hardy, GH, Littlewood, JE, Pólya, G: Inequalities. Cambridge University Press Cambridge (1934)

9. Ghandehari, M: Polar duals of convex bodies. Proc Am Math Soc. 113(3):799-808 (1991)

doi:10.1186/1029-242X-2012-90

Cite this article as: Zhao et al:: Polar duals of convex and star bodies. Journal of Inequalities and Applications 2012 2012:90. 